\title{
2-TO-1 MAPS WITH HEREDITARILY INDECOMPOSABLE IMAGES
}

\author{
JO HEATH \\ (Communicated by Dennis Burke)
}

\begin{abstract}
It is shown that there is no 2-to-1 map from the pseudoarc, or any treelike continuum, onto a hereditarily indecomposable continuum, and that no hereditarily indecomposable treelike continuum can be the image of a 2-to-1 map.
\end{abstract}

\section{INTRODUCTION}

The following are important questions in the study of 2-to-1 maps. The second was asked by J. Mioduszewski in the early 1960's, see [9]; the fourth was asked by S. Nadler and L. E. Ward, Jr. in [10]; the other two are more recent. All four appear in [3], [5], and [6].

(1) Is there a 2-to-1 map defined on any indecomposable arclike continuum?

(2) Is there a 2-to-1 map defined on the pseudoarc?

(3) If the hereditarily indecomposable continuum $Y$ is the image of the pseudoarc under a 2-to-1 map, then must $Y$ be a pseudoarc?

(4) Can any treelike continuum be the image of 2-to-1 map?

We provide a complete answer to (3) and partial answers to the other three. In $\S I V$ Theorem 4 says that there is no 2-to-1 map from the pseudoarc, or any treelike continuum, onto a hereditarily indecomposable continuum, to answer (3) and partly answer (1) and (2). Theorem 3 says that no hereditarily indecomposable treelike continuum can be the image of a 2-to-1 map, to partly answer (4). Theorem 5 also sheds some light on question (2).

A function (called a map if it is continuous) is 2-to-1 if each point inverse has exactly two elements, and a 2-to-1 map is called crisp if the inverse of each proper subcontinuum $C$ of the image is the union of two disjoint continua and $f$ is a homeomorphism from each of them onto $C$. A simple example of a crisp map is the covering map $z^{2}$ from the circle onto itself. In fact, in §III, where the properties of crisp maps are developed, one of the most important of these is that each crisp map is a local homeomorphism. All crisp maps are

Received by the editors October 23, 1989 and, in revised form, December 10, 1990.

1980 Mathematics Subject Classification (1985 Revision). Primary 54C10.

Key words and phrases. Hereditarily indecomposable continua, 2-to-1 map, covering map, pseudoarc, treelike, arclike. 
covering maps then, except that some standard references, Massey's Algebraic Topology for instance, require the domain and image of a covering map to be locally pathwise connected, and the main use of crisp maps in this paper is applications to spaces which are not locally connected. Conversely, Lemma 3 in $\S$ II says that each 2-to-1 covering map has a crisp restriction. As an illustration, the antipodal map on $S^{2}$ is a 2-to-1 covering map that is not crisp, but this map restricted to any equator of $S^{2}$ is the same as the crisp $z^{2}$ map on $S^{1}$.

Suppose $f$ is a crisp map from the continuum $X$ onto $Y$. In $\S$ III we show that $f$ is a local homeomorphism, that $X$ does not have the fixed point property, that $Y$ is not treelike, that $X$ is hereditarily indecomposable iff $Y$ is hereditarily indecomposable, and that $Y$ is indecomposable if $X$ is. We also give an example of a decomposable continuum $X$ and a crisp map from $X$ onto an indecomposable continuum $Y$.

A glossary at the back of the paper gives the definitions of terms used from continuum theory as well as the definitions of special terms described in this paper and in earlier papers on 2-to-1 maps.

\section{WHEN CRISP RESTRICTIONS MUST EXIST}

The existence of a crisp map from $X$ onto $Y$ is a very strong one so it is not surprising that strong assumptions are required. In Lemma 1 we need that $Y$ is hereditarily indecomposable; in Lemma 2 we require (in part) that $X$ is hereditarily indecomposable; and in Lemma 3 we need for $f$ to be open. Since open maps are local homeomorphisms iff they are $k$-to-1 for some integer $k$, see [2] or [11] for example, the hypothesis of open and 2-to-1 is quite strong indeed.

Lemma 1. Each 2-to-1 map from a continuum $X$ onto a hereditarily indecomposable continuum has a crisp restriction to a subcontinuum of $X$.

Proof. Using Zorn's lemma, a minimal continuum $Y$ in the image exists with respect to the property that $f^{-1}(Y)=X^{\prime}$ is connected. (Of course for many maps such a reduced restriction would be degenerate.) A theorem of $\mathrm{H}$. Cook's in [1] says that any map to a hereditarily indecomposable continuum is confluent. So, suppose $C$ is a proper subcontinuum of the image. Then, since $f$ is reduced, $f^{-1}(C)$ is not connected, but since $f$ is confluent, each component of $f^{-1}(C)$ maps onto $C$. Hence, since $f$ is 2-to- $1, f$ is crisp.

Lemma 2. Suppose $f$ is a 2-to-1 map from a hereditarily indecomposable continuum onto $Y$. Then $f$ is crisp iff for each connected nondense subset $D$ of $Y, f^{-1}(D)$ is not connected.

Proof. This lemma is the main theorem in [6].

Lemma 3. Every open 2-to-1 map on a continuum has a crisp restriction to a subcontinuum; in particular, this is true for covering maps.

Proof. We first establish a useful fact used in this proof and later proofs. 
Fact 1. The twin map (the map that takes each $x$ in $X$ to $x^{-}$, the other point of $X$ that maps the same as $x$ ) is continuous iff $f$ is open. Mioduszewski showed in [9] that the twin map is always semicontinuous, i.e. if $\left\{x_{i}\right\}$ converges to $x$ and the twin sequence $\left\{x_{i}\right\}$ converges to the point $p$, then $p$ is either $x$ or $x^{\wedge}$. If the twin function is not continuous, then for some $x, x$ is the limit of two twin sequences and $f$ is not open at $x^{-}$. The converse is equally straightforward.

Now suppose $f$ is a reduced 2-to-1 restriction of an open 2-to-1 map defined on a continuum. Then $f$ is also open and its domain $X$ is a continuum; let $Y$ denote the image of $f$. Now let $S$ be a proper subcontinuum of $Y$. Since $f$ is reduced, $f^{-1}(S)$ is not connected and so it is the union of two disjoint compacta $S 1$ and $S 2$ in $X$. Let $M$ denote the set of points in $S 1$ that map the same as some point of $S 2$. $M$ is not empty since $f(S 1)$ intersects $f(S 2)$. It follows from Fact 1 that $M$ is both open and closed in $S 1$. Likewise the set $N$, of points in $S 2$ that map the same as a point of $S 1$, is open and closed in $S 2$. Note that $f(M)=f(N)$. But $S$ is the union of the three disjoint open sets $f(M), f(S 1 \backslash M)$, and $f(S 2 \backslash N)$, so $S 1 \backslash M$ and $S 2 \backslash N$ are empty. But this means $f$ maps the compactum $M$ 1-to-1 onto $S$, so $f$ is a homeomorphism from the continuum $M$ onto $S$; likewise $f$ is a homeomorphism from $N$ onto $S$.

Because covering maps are local homeomorphisms, they are open, and we have shown there is a crisp refinement.

\section{SOME PROPERTIES OF CRISP MAPS}

Theorem 1. Every crisp map on a continuum is a local homeomorphism.

Proof. Suppose $f$ is a crisp map from the continuum $X$ onto $Y$. Then $f$ is 2-to-1 and it was shown in Theorem 1 in [4] that there are disjoint twin open sets ( $t$ win sets are defined in the glossary) in $X$. Hence there are twin open sets $V$ and $V^{\wedge}$ in $X$ with disjoint closures. If $f$ is not a local homeomorphism, then there is a hinge point $z$ in $X$. That is, there are twin sequences, $\{p(i)\}$ and $\left\{p(i)^{\wedge}\right\}$, that both converge to $z$. We may assume that no $p(i)$ or $p(i)^{\wedge}$ is in $V \cup V^{\wedge}$, since $z$ cannot be in the closures of both $V$ and $V^{\wedge}$. For each $i$, let $C(i)$ be the component of $X \backslash\left(V \cup V^{\wedge}\right)$ containing $p(i)$. Since $X$ is a continuum, each $C(i)$ must "go to the boundary," see [7] for instance, so there is for each $i$ a point $x(i)$ in $C(i) \cap \bar{V}$ or in $C(i) \cap \bar{V}^{\wedge}$; we assume the former for each $i$. Since $f(C(i))$ is not all of $Y(f(V)$ is in $Y \backslash f((C(i))$ for instance), $[C(i)]^{\wedge}$ is a continuum disjoint from $C(i)$ that contains $p(i)^{\wedge}$ and $x(i)^{\wedge}$, by the crispness of $f$. By taking subsequences if necessary, we have $\{C(i)\}$ converging to a continuum $C$ that contains $z$ and the limit $x$ of the sequence $\{x(i)\}$ from $\bar{V}$; and we have $\left\{C(i)^{\wedge}\right\}$ converging to a continuum $K$ that contains $z$ and the limit $x^{\wedge}$ of the sequence $\left\{x(i)^{\wedge}\right\}$ from $\bar{V}^{\wedge}$. But if $z$ is in $C \cap K$ then $C \cup K$ is a continuum; if $V \cup V^{\wedge}$ misses $C \cup K$ then $C \cup K$ does not map onto $Y$; and if $\left\{x, x^{\wedge}\right\}$ is in $C \cup K$ then $C \cup K$ intersects its twin. This contradicts the definition of crisp. 
The following corollary might be compared with I. Rosenholtz's theorem in [11], which says that each local homeomorphism defined on a chainable continuum is a homeomorphism (and so, of course, is not 2-to-1).

Corollary 1. If $f$ is a crisp map on $X$ then $X$ does not have the fixed point property.

Proof. If $f$ is crisp, it is a local homeomorphism, and hence open. As was shown in Fact 1 in the proof of Lemma 3, the twin map is continuous, and of course the twin function has no fixed point.

Theorem 2. There is no crisp map from a continuum onto a treelike continuum.

Proof. On the contrary suppose that $f$ is such a map. Since $f$ is a local homeomorphism, there is a positive number $e$ such that for each $x$ in $X$, the distance from $x$ to $x^{-}$is greater than $e$.

For each point $q$ in $Y$, let $W(q)$ and $W(q)^{\wedge}$ denote disjoint twin open sets in $X$, each of diameter less than $e / 4$, that contain the two inverse points of $q$, and let $U(q)$ denote the open set $f(W(q))$. Now cover $Y$ with these $U(q)$ neighborhoods; there is a tree shaped finite refinement $T$ covering $Y$, $C(1), C(2), \ldots, C(m)$. Being tree shaped implies that no subcollection of $T$ with at least three elements forms a circular chain. For each $i, C(i)$ is in some $U(q)$ so the inverse of $C(i)$ is the union of two disjoint twin open sets, $D(i)$ and $D(i)^{\wedge}$, each of diameter less than $e / 4$. Let $D$ denote $\{D(i): i=$ $1,2, \ldots, m\} \cup\left\{D(i)^{\wedge}: i=1,2, \ldots, m\right\}$, a cover of $X$.

We show that $X$ is not connected for a contradiction. Choose a particular point $p$ of $X$ and define $E$ to be the set of points $x$ in $X$ such that both $p$ and $x$ belong to the union of a coherent subcollection of $D$. Suppose some twin pair of points, $x$ and $x^{\wedge}$, both belong to $E$. Some minimal coherent subcollection of $D$ has both $x$ and $x^{\wedge}$ in its union. This subcollection forms a chain, $C$, and we can assume that no proper subchain contains a twin pair of points. The chain $C$ must have at least four links because each link has diameter less than $e / 4$ and $x$ and $x^{\wedge}$ cannot be within $e$ of each other. Because no two nonadjacent interior links of $C$ contain twin pairs of points, their images do not intersect. Hence, since the images of the first and last links of $C$ do intersect, the image of $C$ is a circular chain in $T$. This contradicts the assumption that $x$ and $x^{-}$both belong to $E$. Each point of $E$ then is in an open set (from $D$ ) that misses $E^{\curlywedge}$, and vice-versa; hence $X=E \cup E^{\wedge}$, two disjoint open sets.

Notation. If $x$ is a point of a continuum $X$, let $C(x, X)$ denote the composant of $X$ based on $x$.

Lemma 4 (Some easy basic facts). If $f$ is a crisp map from the continuum $X$ onto $Y$ then:

(1) if $X=A \cup B$, two continua, then one of them maps onto $Y$; 
(2) if $C$ is a proper subcontinuum of $X$ that maps onto $Y$ then $X=$ $C \cup C^{\wedge}$, two proper subcontinua of $X$;

(3) $\left(C\left(x^{\wedge}, X\right)\right)^{\wedge}=C(x, X)$ and $C(f(x), Y) \subseteq f(C(x, X))$; and

(4) if $X$ is indecomposable, then so is $Y$, and $f$ is 2-composant-to- 1.

Proof. (1) If $f(A)$ is a proper subcontinuum of $Y$ then $f^{-1}(f(A))$ is the union of two disjoint twin continua $A \cup A^{\wedge}$. Then $A^{\wedge}$ is contained in $B$ so $f(B)$ contains $f\left(A^{\wedge}\right)=f(A)$. Hence $f(B)=Y$.

(2) $C^{\wedge}$ is a continuum since the twin map is continuous (see Fact (1) in the proof of Lemma 3). It is a proper subset of $X$ since $C \subseteq C^{\wedge}$ implies $C^{\wedge} \subseteq C$, and if $f(C)=Y$ then every point of $X$ is either in $C$ or $C^{\wedge}$.

(3) If $y$ belongs to $C(x, X)$ and $M$ is a proper subcontinuum of $X$ containing $x$ and $y$, then (i) if $f(M)=Y$, it follows from (2) that $M^{\wedge}$ is a proper subcontinuum of $X$ containing $y^{\wedge}$ and $x^{\wedge}$ and (ii) if $f(M)$ is a proper subcontinuum of $Y$ then $M^{\wedge}$ is again a proper subcontinuum of $X$ since $f$ is crisp. The proof of the inclusion is even more straightforward.

(4) Since $X$ is indecomposable, there are infinitely many disjoint sets of the form $f(C(x, X))$ in $Y$, each containing a composant of $Y$ (from part (3)) so $Y$ is indecomposable. To see that $f$ is 2-composant-to-1 we need, in view of (3) again, only that $f(C(x, X))$ is contained in $C(f(x), Y)$ for each $x$ in $X$. So suppose $z$ is a point of $f(C(x, X))$. Then some $z^{\prime}$ in $C(x, X)$ maps to $z$, and $z^{\prime}$ is in a proper subcontinuum $E$ of $X$ containing $x$. If $f(E)$ is not all of $Y$ then $f(E)$ is a proper subcontinuum of $Y$ containing $z$ and $f(x)$, so $z$ belongs to $C(f(x), Y)$ as desired. So suppose $f(E)=Y$. From (2) we infer that $X=E \cup E^{\wedge}$, contradicting the fact that $X$ is indecomposable.

Corollary 3. If $f$ is a crisp map from the continuum $X$ onto $Y$, then $X$ is hereditarily indecomposable iff $Y$ is hereditarily indecomposable.

Proof. If $X$ is hereditarily indecomposable, then $Y$ is indecomposable by Lemma 4(4), and each proper subcontinuum in $Y$ is indecomposable since $f$ is crisp.

Now suppose $Y$ is hereditarily indecomposable and some proper subcontinuum of $X$ maps onto $Y$. If $C$ denotes an irreducible continuum in $X$ that maps to $Y$, then from Lemma 4(2) we see that $X=C \cup C^{\wedge}$, two proper subcontinua of $X$ that each map onto $Y$. Let $V$ denote an open set whose closure is in $C \backslash C^{\wedge}$; then $V^{-}$is an open set whose closure is in $C^{\wedge} \backslash C$. Let $x$ denote a point common to $C$ and $C^{\wedge}$, and let $D$ and $E$ denote the components of $C \backslash V$ and $C^{\wedge} \backslash V^{\wedge}$, respectively, containing $x$. But since $D \cup E$ does not map onto $Y$, no two points of $D \cup E$ map the same, so $f(D) \cup f(E)$ is a decomposable subcontinuum of $Y$. This contradiction means that no proper subcontinuum in $X$ maps onto $Y$. But then $X$ is indecomposable by Lemma 4(1). If $M$ is a proper subcontinuum of $X$, then $M$ is homeomorphic to $f(M)$ and so is indecomposable. Hence $X$ is hereditarily indecomposable.

Although we have seen that crisp maps preserve indecomposability and hered- 


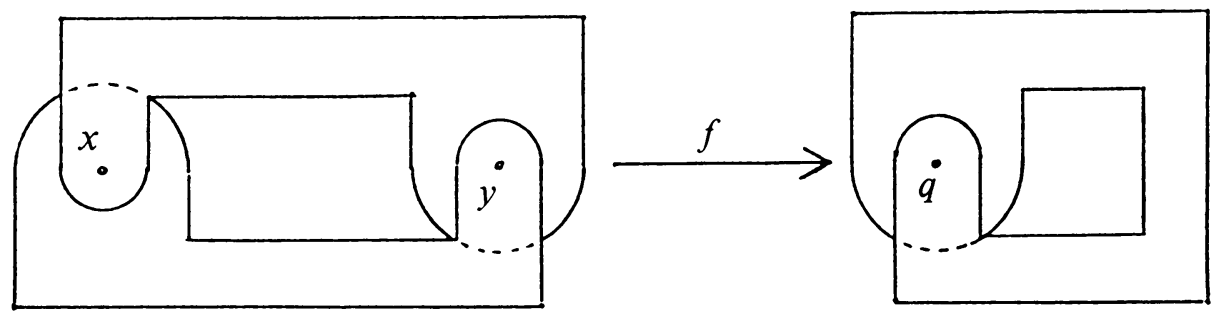

FIGURE 1

itary indecomposability, they do not preserve decomposability (see Figure 1).

Example. A decomposable continuum $X$ and a crisp map $f$ from $X$ onto an indecomposable continuum $Y$.

Description. Let $H$ denote an indecomposable continuum. Let $p$ and $t$ denote two points of $H$ from different composants. Let $H 1$ and $H 2$ denote two disjoint copies of $H$ and let $p 1$ and $t 1$ in $H 1$ and $p 2$ and $t 2$ in $H 2$ be the copies of $p$ and $t$. Now, to get $X$, sew $H 1$ to $H 2$ at two points. Attach $t 1$ to $p 2$ to get a point $x$ in $X$, and attach $t 2$ to $p 1$ to get another point $y$ in $X$. Let $Y$ be the continuum $H$ with the two points $p$ and $t$ identified as a single point $q$. To describe $f$, first let $h 1$ denote a homeomorphism from $H 1$ onto $H$ that takes $p 1$ to $p$ and $t 1$ to $t$ and similarly let $h 2$ denote a homeomorphism from $H 2$ onto $H$ that takes $p 2$ to $p$ and $t 2$ to $t$. Then define $f$ so that $f$ restricted to $X \backslash\{x, y\}$ is $h 1 \cup h 2$ restricted to $(H 1 \backslash\{p 1, t 1\}) \cup(H 2 \backslash\{p 2, t 2\})$ and so that $f(x)=f(y)=q$.

\section{THE RESULTS}

These theorems come quickly from the other theorems in this paper and some reference theorems.

Theorem 3. There is no 2-to-1 map defined on a continuum whose image is a hereditarily indecomposable treelike continuum.

Proof. Suppose there is such a map. From Lemma 1 there is a crisp restriction to a subcontinuum and the image of this restriction is still a hereditarily indecomposable treelike continuum. This contradicts Theorem 1.

Theorem 4. There is no 2-to-1 map from a treelike continuum onto a hereditarily indecomposable continuum.

Proof. Suppose there is such a map. Once again there is a crisp restriction to a subcontinuum (still treelike) of $X$ onto a continuum that is hereditarily indecomposable. But crisp maps are confluent and McLean has shown [8] that the confluent image of a treelike continuum is a treelike continuum. This contradicts Theorem 3 .

The following theorem says more than the 2-to-1 image of a pseudoarc can not be hereditarily indecomposable; somehow the civilized behavior of $E$ is 
incongruous with its being a 2-to-1 image of the pseudoarc, but the author is not sure why (yet).

Theorem 5. Suppose there is a 2-to-1 map defined on the pseudoarc. Then there is a subcontinuum $E$ of the image and a connected set $D$ in the image such that (1) $E=\bar{D} \cup F$, two proper subcontinua of $E$ such that $D$ misses $F$ and (2) the inverse of $D$ is connected.

Proof. Suppose there is a 2-to-1 map on the pseudoarc. As was briefly argued in the proof of Lemma 1 , there is a reduced restriction $f$ to a subpseudoarc that maps onto a continuum $Y$. By Corollary $3, Y$ is hereditarily indecomposable if $f$ is crisp and this contradicts Theorem 4. So, since $f$ cannot be crisp, it follows from Lemma 2 that there is a connected nondense subset $D$ of $Y$ such that $f^{-1}(D)$ is connected (but $f^{-1}(\bar{D})$ is not connected since $f$ is minimal). Let $Z$ denote the component of $f^{-1}(\bar{D})$ that contains $f^{-1}(D)$, and let $W$ denote any other component. There is a continuum $W^{\prime}$ containing $W$ and some point not in $f^{-1}(\bar{D})$ such that $W^{\prime}$ misses $Z$. Then $E=\bar{D} \cup f\left(W^{\prime}\right)$, where $F$ is $f\left(W^{\prime}\right)$, satisfies the theorem.

\section{GLOSSARY}

1. A continuum $X$ is arclike if for each positive number $\varepsilon$, there is an $\varepsilon$-map (each point inverse has diameter less than $\varepsilon$ from $X$ onto the interval $[0,1]$.

2. A chain is a finite collection of sets $C 1, C 2, \ldots, C n$ such that $C i$ intersects $C j$ iff $|i-j| \leq 1$.

3. A circular chain is a finite collection of sets $C 1, C 2, \ldots, C n$ such that $C i$ intersects $C j$ iff $|i-j| \leq 1$ or $C i$ and $C j$ are the first and last terms.

4. A collection $G$ of sets is coherent if for any two elements $g$ and $g^{\prime}$ of $G$ there is a finite sequence $g_{1}, g_{2}, g_{3}, \ldots, g_{n}$ of elements of $G$ such that $g=g_{1}, g^{\prime}=g_{n}$, and for each relevant $i, g_{i}$ and $g_{i+1}$ intersect in a nonempty set.

5. A map $f$ is confluent if for each continuum $C$ in the image, each component of the inverse of $C$ maps onto $C$.

6. The subset $M$ of a continuum $X$ is a composant of $X$ if there is a point $x$ in $X$ such that $M$ is the set of all points of $X$ contained in a proper subcontinuum of $X$ containing $X$. If $X$ is indecomposable, then $X$ has uncountably many disjoint composants.

7. A space is a continuum if it is both connected and compact. (In this paper it is also assumed to be metric.)

8. A map $f$ is crisp if the inverse of each proper subcontinuum is the union of two disjoint continua and $f$ is 1-to-1 on each.

9. A $k$-to-1 map on a compactum is a covering map if it is a local homeomorphism. Some definitions also require the domain and range to be locally pathwise connected.

10. A continuum is decomposable if it is the union of two proper subcontinua. 
11. A space $X$ has the fixed point property if every continuous map from $X$ into itself has a fixed point.

12. A continuum is hereditarily indecomposable if each of its subcontinua is indecomposable.

13. A point $p$ in the domain of a 2-to-1 map is a hinge point if there are twin sequences $\left\{x_{i}\right\}$ and $\left\{\hat{x_{i}}\right\}$ that both converge to $p$. The " $\wedge$ " notation is defined in 18.

14. A continuum is indecomposable if it is not the sum of two of its proper subcontinua.

15. A map $f$ is a local homeomorphism if for each point $p$ in the domain, there is an open set $U$ containing $p$ such that $f$ is a homeomorphism on $U$ and $f(U)$ is open.

16. A map is reduced if each proper subcontinuum in the image has disconnected preimage.

17. A continuum $X$ is treelike if for each positive number $\varepsilon$ there is an $\varepsilon$-map from $X$ onto a tree, i.e. a connected acyclic finite graph.

18. If $f$ is a 2-to-1 map defined on $X$, then the twin map is the map that takes each point $x$ in $X$ to $x^{\wedge}$, the unique point such that $f(x)=f\left(x^{\wedge}\right)$.

19. $A$ and $B$ in $X$ are twin sets if $B=A^{\wedge}=\left\{a^{\wedge}: a \in A\right\}$. The twin $a^{\wedge}$ of $a$ is defined in 18 .

\section{REFERENCES}

1. H. Cook. Continua which admit only the identity map onto nondegenerate subcontinua, Fund. Math. LX (1967), 241-249.

2. S. Eilenberg, Sur quelques proprietes des transformations localement homeomorphes, Fund. Math. V24 (1935), 35-42.

3. Jo Heath, Treelike continua and exactly $k$-to-1 functions, Proc. Amer. Math. Soc. 105 (1989).

4. $\_$, The structure of 2-to- 1 maps on metric compacta, Proc. Amer. Math. Soc. 110 (1990), 549-555.

5. __ Four key questions in the theory of 2-to- 1 maps, Colloq. Math. Soc. János Bolyai, Topology, Pécs (Hungary), 1989.

6. __ K-to- 1 maps on hereditarily indecomposable metric continua, Trans. Amer. Math. Soc. (to appear).

7. C. Kuratowski, Topologie, I, II, Monogr. Mat., Warszawa, Wroclaw, 1948, 1950.

8. T. Bruce McLean, Confluent images of treelike curves are treelike, Duke J. Math. 39 (1972), $465,473$.

9. J. Mioduszewski, On two-to-one continuous functions, Dissertationes Math. 24 (1961), 42.

10. S. B. Nadler, Jr. and L. E. Ward, Jr., Concerning exactly $(n, 1)$ images of continua, Proc. Amer. Math. Soc. 87 (1983), 351-354.

11. I. Rosenholtz, Open maps of chainable continua, Proc. Amer. Math. Soc. 42 (1974), 258264. 